\title{
Atrial Fibrillation in cryptogenic stroke The Nordic Atrial Fibrillation and Stroke Study (NOR-FIB)
}

Anna Tancin Lambert ${ }^{2,3}$, Xiang Yi Kong 3 , Barbara Ratajczak-Tretel2,3, David Russell',3,Vigdis Bjerkeli', Mona Skjelland', Halvor Næss ${ }^{5}$, Else Charlotte Sandset ${ }^{1}$, Hege $^{2}$ Ihle-Hansen ${ }^{3}$, Titto Idicula ${ }^{6}$, Håkon Tobro ${ }^{7}$, Siv B. Krogseth ${ }^{8}$, Håkon Ihle-Hansen 9 , Guri Hagberg ${ }^{9}$, Kathrine Arntzen ${ }^{10}$, Grete Kristin Bakkejord ${ }^{10}$, Thomas C. Truelsen ${ }^{11}$, Karen L. / Agidius ${ }^{12}$, Christina Kruuse ${ }^{13}$, Maja Villseth ${ }^{14}$, Guttorm Eldøen ${ }^{15}$, Raheel Shafiq ${ }^{15}$, Anne Kristine Gulsvik ${ }^{16}$, Håkon Lerstad ${ }^{16}$, Anita Vatne ${ }^{17}$, Martin Kurz $^{18}$, Jesper Sømark ${ }^{19}$, Susanne Ingebrigtsen ${ }^{20}$, Eric Schordan ${ }^{21}$, Hüseyin Firat ${ }^{21}$, Gudrun A. Høie ${ }^{22}$, Dan Atar ${ }^{3,4}$, Bente E. Halvorsen ${ }^{3}$, Anne Hege Aamodt 'Department of Neurology, Oslo University Hospital, Norway, ${ }^{2}$ Department of Neurology, Østfold Hospital Trust, Norway, ${ }^{3}$ Institute of Clinical Medicine, University of Oslo, Norway, ${ }^{4}$ Department of Cardiology, Oslo University Hospital, Norway, ${ }^{5}$ Department of Neurology, Haukeland University Hospital, Norway, ${ }^{6}$ Department of Neurology, St. Olav Hospital, Norway, ${ }^{7}$ Department of Neurology, Telemark Hospital, Norway, ${ }^{8}$ Department of Neurology, Vestfold Hospital, Norway, ${ }^{\circ}$ Department of Internal Medicine, Vestre Viken Hospital Trust, Norway, ${ }^{10}$ Department of Neurology, Norland Hospital, Norway, " Department of Neurology, Rigshospitalet, Copenhagen, Denmark, ' ${ }^{2}$ Department of Neurology, Bispebjerg Hospital, Denmark, ${ }^{3}$ Department of Neurology, Herlev Gentofte Hospital, Denmark, ${ }^{14}$ Department of Neurology, Vestre Viken Hospital Trust, Norway, ${ }^{15}$ Department of Neurology, Molde Hospital, Møre og Romsdal Hospital Trust, Norway, ${ }^{16}$ Department of Internal Medicine, Diakonhjemmet Hospital, Norway, ${ }^{17}$ Department of Neurology, Sørlandet Hospital Kristiansand, Norway, ${ }^{18}$ Department of Neurology, Stavanger University Hospital, Norway, ${ }^{19}$ Department of Neurology, Lillehammer, Innlandet Hospital Trust, Norway, ${ }^{20}$ Department of Neurology, University Hospital of North Norway, Norway, ${ }^{21}$ Firalis SA, Biomarker R\&D, Huningue, France, ${ }^{22}$ Department of Cardiology, Østfold Hospital Trust, Norway

\section{Background}

Relatively large group of ischemic strokes or TIAs, up to $40 \%$, remains without a clear etiology, cryptogenic stroke / TIA. Almost one fourth of these patients have a silent atrial fibrillation that remains undiscovered. Insertable cardiac monitors (ICMs) show higher detection rates of atrial fibrillation (AF) compared to standard clinical follow-up. Identification of reliable biomarkers indicating $A F$ will be valuable in the selection of patients for prolonged cardiac rhythm monitoring.

\section{Objective:}

- To assess the incidence of AF detection using ICM (Reveal LINQ尺) in patients with cryptogenic stroke / TIA.

- To identify biomarkers that can be used as predictors of incident AF Study design: International multi-center prospective observational study of the occurrence of AF in cryptogenic stroke / TIA patients with ICMs for 12 months. Blood samples measuring biomarkers are taken in the acute phase and at 12 months' follow-up. Inclusion of patients started in January 2017. Study duration until December 2019. Estimated number of patients 500 .

Primary endpoint: AF detection rate within 12 months

Secondary endpoints: AF detection rate within 6 months. Levels of cardiovascular biomarkers at baseline and 12 months

Figure I. Flow chart of the main study

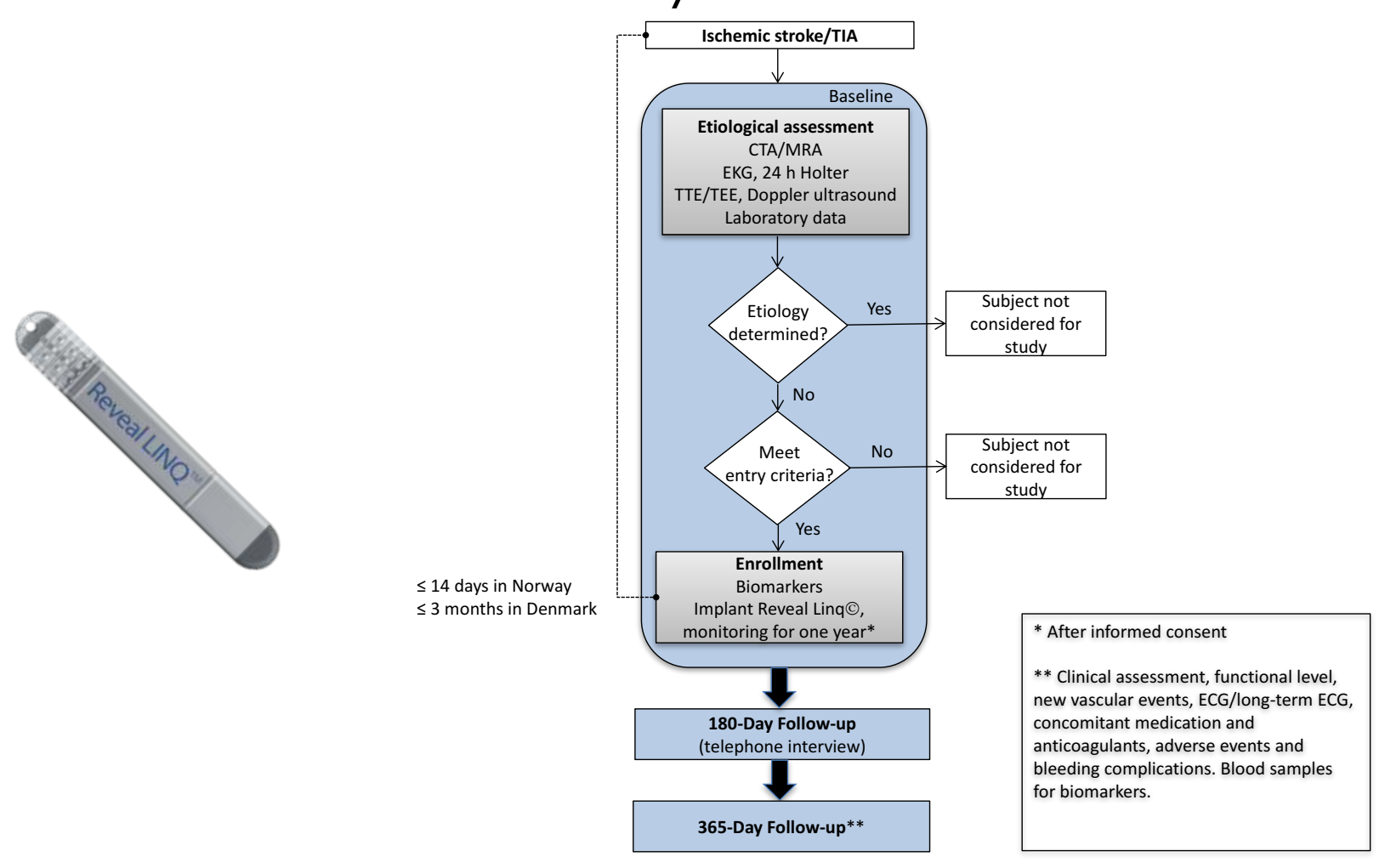

Preliminary results

By October 2018, the total number of patients included in 13 out of 19 participating centres is II5. AF / atrial flutter has been detected in 26 patients, resulting in detection rate of $22,6 \%$.

Biomarker analysis in the pilot study

Blood samples from 3 groups of participants:

- 9 patients with ischemic stroke / TIA due to AF (AF group)

- 8 patients with ischemic stroke / TIA due large artery atherosclerosis with carotid stenosis (ATS group)

- 10 age and sex-adjusted controls (Control group)

Analyses with the Olink Proseek Multiplex Cardiovascular 96x96 kit measuring together 184 cardiovascular disease related human protein biomarkers with reagents based on Proximity Extension Assay technology were carried out. Non-parametric Mann-Whitney $U$ test was used to determine statistical difference between AF and ATS group, and AF and control group.
Figure 2. Median LOG 2 levels of the II most significant biomarkers in the different groups of participants

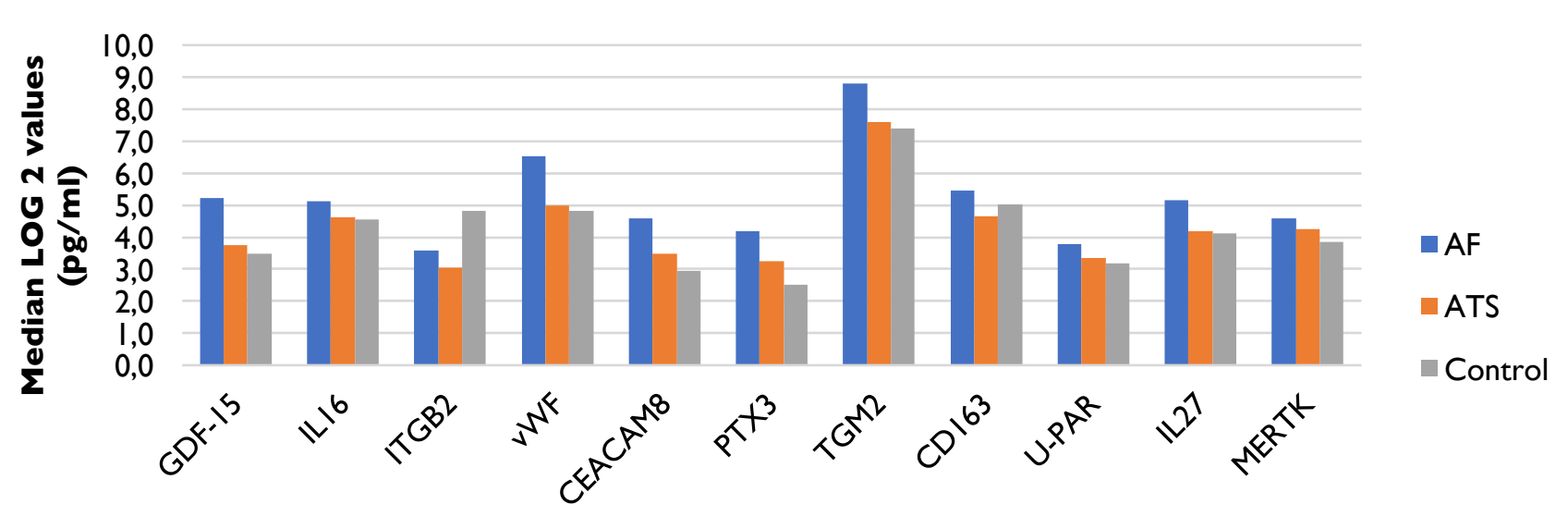

Biomarkers

For further testing Geneset enrichment analysis (GSEA) was applied to our dataset against the Gene Onthology Consortium database (c5.all.v6.I) downloaded from MiSigDB (http://www.broadinstitute.org/gsea/msigdb/).

We used phenotype permutation for 1000 times, and chose the T-test statistical approach to rank genes and complete the GSEA analysis.

Figure 3. GSEA, enrichment plots (A) of significantly enriched gene-sets and heat-map (B) representation of the top 50 positively correlated proteins to the $A F$ group
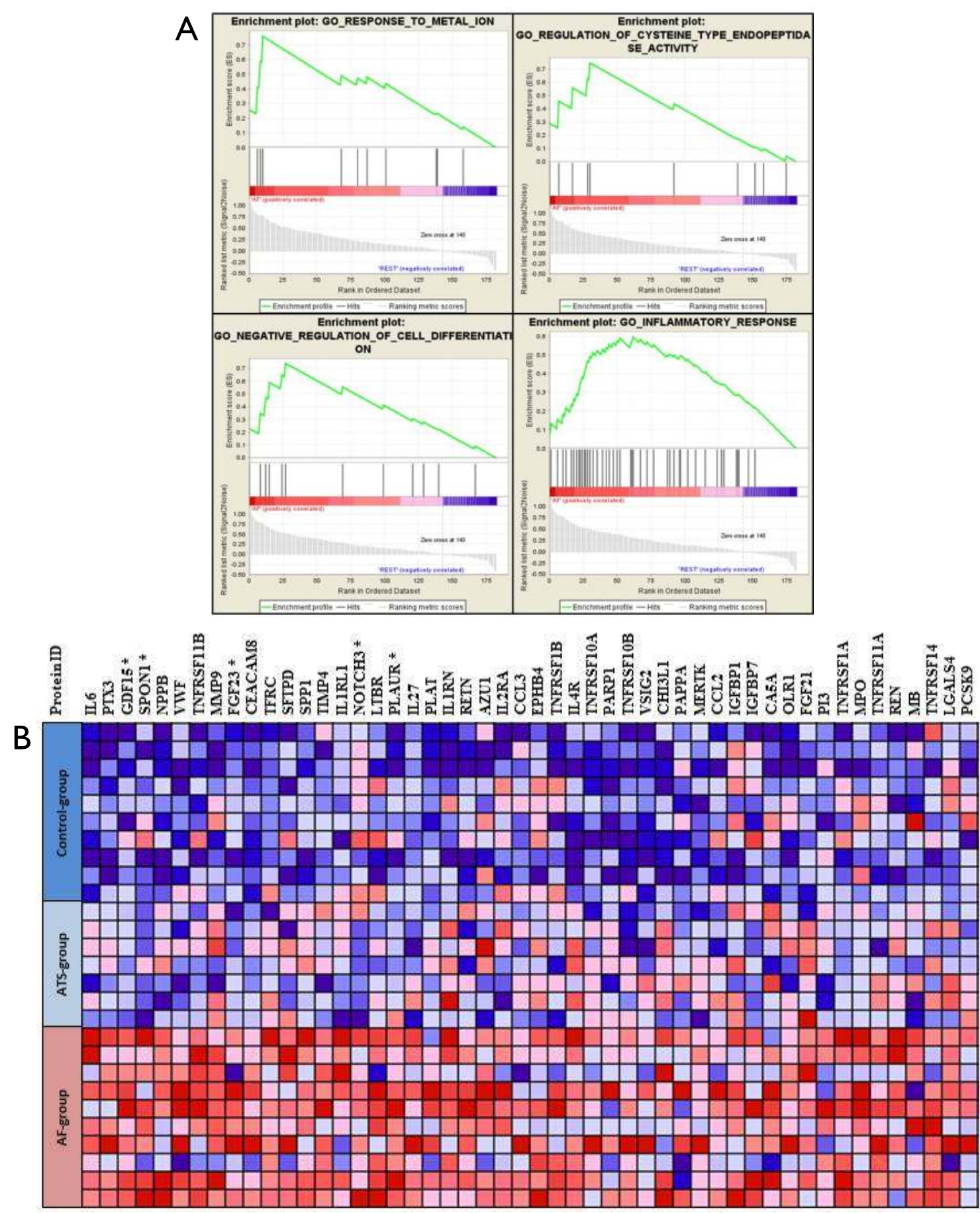

Conclusion

ICMs are effective in detecting AF in patients with cryptogenic stroke. There are multiple proteins that may potentially be used as biomarkers for the detection of AF in cryptogenic stroke / TIA patients. However, studies with larger samples are needed. In the on-going NOR-FIB study we plan further biomarker assessment in patients with cryptogenic stroke / TIA undergoing long-term cardiac rhythm monitoring with ICM. 